We conducted a systematic review and meta-analysis, searching six databases and including original research manuscripts comparing testing outside clinics with conventional testing. Main outcomes were test uptake and linkage to care, delivery models and adverse outcomes.

We identified 76 studies which were included. Data from 18 studies were pooled in meta-analyses. Studies focused on HIV (48), chlamydia (8), and multiple diseases (20). HIV selftesting increased test uptake compared with facility-based testing (nine studies, pooled OR $2.59,95 \% \mathrm{CI}=1 \cdot 06-6 \cdot 29$, moderate quality). STI self-sampling increased test uptake compared with facility-based testing (seven studies, pooled OR $1.7495 \% \mathrm{CI}=0.97-3.12$, moderate quality). Innovative and other digital test delivery services improved test uptake compared with facility-based testing services. We defined digital as emails, websites, instant messaging, or related internet approaches.

Our work shows testing outside of clinics increased test uptake without significant adverse outcomes. These testing approaches provide an opportunity to expand access and empower patients through innovative delivery models. From the policy viewpoint, this work demonstrates that the use of digital technology in testing outside clinics is a useful strategy for improving infectious diseases screening and linkage to care, and policies that aim to promote the use of these strategies are needed. These models may be especially useful for diseases associated with stigmatization such as HIV and other STIs in LMICs. However, inherent challenges include ensuring linkage to care continuum, quality assured testing and getting the right tests to the right people. Further implementation research and scale-up of effective decentralized models in LMIC settings is needed.

\section{S08.2 SELF-TESTING AND E-STI: THE DUTCH EXPERIENCE WITH HOME-BASED AND INTERNET-BASED SERVICES AND TRANSFORMATION INTO EFFECTIVE IMPLEMENTATION}

E Op de Coul*. Rivm, Bilthoven, The Netherlands

10.1136/sextrans-2021-sti.38

The main STI-healthcare providers in the Netherlands are GPs and sexual health clinics (SHCs). SHCs offer free-of-charge STI-care for key-populations. At the same time, Internet-based STI-test and home-sampling offers increase, offering new opportunities for a blended, more efficient, low threshold service. Currently, SHCs and national partners are reshaping sexual healthcare for young people and other key-populations, using a stepped-care model (SCM). The SCM is based on the principal that not all individuals need the same level of STIcare: some may be helped by online self-assessment tools (sense.info, Advies.chat) and a home sampling-kit and others need support by a professional.

NoMoreC and Limburg4zero are regional e-STI services for MSM, developed by SHCs, and co-created with MSM communities, healthcare professionals and other stakeholders, based on a scientific/theoretical framework. NoMoreC targets high-risk MSM for HCV-prevention and offers anonymous, low cost, home-sampled $\mathrm{HCV}$-testing. Limburg4zero provides home-sampling for HIV/STI testing as an extension of regular STI-care including peer-to-peer sharing, and e-counselling on e.g. chemsex and PrEP.
Opportunities

- Community involvement is essential to reach the right target groups

- Personalized e-STI advice and test-options provide a viable alternative for those preferring autonomic testing, and can saves costs

- The HCV-test service appeared especially suitable for MSM using PrEP who arrange their own STI-care

- e-STI should deliver prevention messages for those who tested negative

Challenges

- Quality assurance of test procedures (e.g. quality of the test, which test is offered) is a topic that should be high on the agenda

- Implementation is only effective when based on a clear theoretical/scientific framework

- Sustainability of regional e-STI initiatives is challenging; most are financed by research grants and broader/sustainable expansion may need governmental funding

- Tracking linkage-to-care is challenging in interventions with anonymous participation

- Return rates of home-sampling kits may be improved through digital innovations

\section{S08.3a HOW TO REALIZE IMPLEMENTATION IN HIV SELF- TESTING BY DISTRIBUTION VIA THE SOCIAL NETWORK IN CHINA: A JOINED COLLABORATION BETWEEN STAKEHOLDERS}

${ }^{1}$ W Tang ${ }^{*},{ }^{2} Y$ Zhou. 'University of North Carolina Project-China, Guangzhou, China; ${ }^{2}$ Zhuhai Center for Diseases Control and Prevention, Zhuhai, China

10.1136/sextrans-2021-sti.39

HIV self-testing (HIVST) had been widely used in China since 2012, but social network strategies were rarely used to improve HIVST coverage. This presentation reports a case of community-based social network distribution of HIVST among Chinese men who have sex with men (MSM).

In 2016, UNC Project-China hosted an HIV testing intervention development designathon and invited eight teams from two provinces to contribute. The Zhuhai team designed an HIVST promotion proposal, which was a joint working plan between a community-based organization and the local CDC. They codeveloped a social media platform for self-test kits application and results reporting, which successfully provided 600 kits to MSM in 2016. However, due to the lack of evidence-based intervention, the program failed to increase the testing coverage and lack of innovation. After that, the researchers in the UNC Project-China team brought in secondary distribution idea, which allows the index MSM to apply for multiple kits and distribute them to people in their social network. After the piloting, 373 index MSM successfully motivated 287 alters for testing, among whole $40 \%$ were new testers, and the HIV testing yield among alters was significantly higher than that among index MSM. In the following RCT, a monetary incentive and peer referral further improved the mean number of kits that were distributed by index MSM $(\mathrm{MD}=1 \cdot 21,95 \% \mathrm{CI}: 0.77-1 \cdot 65)$. The strategy strongly increased testing coverage and was further implemented at the provincial level.

Opportunities

- Social-media platform provided an opportunity to reach a diverse index MSM 